\title{
FENCHEL EQUALITIES AND BILINEAR MINMAX EQUALITIES
}

\author{
G. H. GRECO, A. FLORES-FRANULIČ, and H. ROMÁN-FLORES*
}

(Dedicated to memory of Werner Fenchel on the occasion of the 100th anniversary of his birth)

\begin{abstract}
Chief objects here are pairs $(X, F)$ of convex subsets in a Hilbert space, satisfying the bilinear minmax equality

$$
\inf _{x \in X} \sup _{y \in F}\langle x, y\rangle=\sup _{y \in F} \inf _{x \in X}\langle x, y\rangle .
$$

Specializing $F$ to be an affine closed subspace we recover and restate crucial concepts of convex duality, revolving around Fenchel equalities, biconjugation, and inf-convolution. The resulting perspective reinforces the strong links between minmax, set-theoretic, and functional aspects of convex analysis.
\end{abstract}

Let $E$ be a (real) Hilbert space with scalar product $\langle\cdot, \cdot\rangle$. Select two convex subsets $X, Y$ of $E$. The relation

$$
\inf _{x \in X} \sup _{y \in Y}\langle x, y\rangle=\sup _{y \in Y} \inf \langle x, y\rangle
$$

is called a bilinear minmax equality with respect to the pair $(X, Y)$. Correspondingly, whenever (1) holds, the couple $(X, Y)$ is said to be a minmax convex pair. Such pairs were introduced and studied in [3], and some criteria for recognizing them were given there.

Von Neumann's theorem [6] about equilibrium in zero-sum two-person games can be restated as "every couple of simplices is a minmax pair". Similarly, Fenchel's theorem on linear programming duality [2] can be restated as "every feasible couple of polyhedral convex sets is a minmax pair".

Motivation for exploring minmax equalities comes from their frequent occurrence and wide applicability. To wit, they may help in modeling zero-sum two-persons games, facilitate the study of inequality systems in terms of alternative formulations, describe or indicate when commutation between universal

\footnotetext{
* This research was undertaken at the UTA (Universidad de Tarapacá) in August 2000. Financial support from Fondecyt-Chile N.1000463 is gratefully acknowledged.

Received April 7, 2005.
} 
and existential quantifiers are allowed, and much more, as we will see in the next section.

Our main Theorem 0.1 characterize minmax pairs $(X, F)$ of a nonempty convex subset $X$ and a closed affine subspace $F$ of a Hilbert space $E$.

For the statement, given a closed affine subspace $F$ of a Hilbert space $E$, denote by $e_{F}$ the point of $F$ nearest to the origin. Let $\mathrm{pr}_{0}$ denote the orthogonal projection of $E$ on the closed vector subspace $F_{0}:=F+\operatorname{R} e_{F}$. Moreover, denote by $Y^{\infty}$ the asymptotic cone $\{v \in E: Y+v \subset Y\}$ of an arbitrary closed nonempty convex subset $Y$ of $E$.

When $F$ is a closed vector subspace (i.e. $\left.e_{F}=0\right), "(X, F)$ is a minmax pair if and only if either $X \cap F^{\perp} \neq \varnothing$ or $\operatorname{dist}\left(X, F^{\perp}\right) \neq 0$ ” [3], where $\operatorname{dist}(X, F):=\inf \{\|x-y\|: x \in X, y \in F\}$. Otherwise,

THEOREM 0.1 (Minmax convex pairs and affine subspaces). Assume $e_{F} \neq 0$. Then $(X, F)$ is a minmax pair if and only if the following two conditions hold:

(2) $\inf \left\{\lambda \in \mathrm{R}: \lambda e_{F} \in \operatorname{pr}_{0} X\right\}=\inf \left\{\lambda \in \mathrm{R}: \lambda e_{F} \in \overline{\operatorname{pr}_{0} X}\right\} \quad$ (inf-normality)

(3) either $\overline{\operatorname{pr}_{0} X} \cap \operatorname{R} e_{F} \neq \emptyset \quad$ or $\quad-e_{F} \notin{\overline{\operatorname{pr}_{0} X}}^{\infty} \quad$ (feasibility).

In the next section 1 Theorem 0.1 is proved. In the last sections $2-3$, we explore and comment relationships between minmax pairs, Fenchel equalities and biconjugation formulae; we will show that the roles played by these three types of equalities are interchangeable.

In the sequel "convex set" always stands for "convex subset of a Hilbert space E", if not otherwise explicitly specified. Some terms coming from convex analysis (e.g. normality, feasibility) will be used as meta-terms for threading similar properties.

Here and in the sequel we adopt Moreau's convention $(+\infty)+(-\infty):=$ $(-\infty)+(+\infty):=+\infty$ together with inf $\emptyset:=+\infty$ and $\sup \emptyset:=-\infty$. This convention assures that $\inf (a+A)=a+\inf A$, for every $a \in \mathrm{R}$ and $A \subset \overline{\mathrm{R}}$. $\overline{\mathrm{R}}$ denotes the extended real line $\mathrm{R} \cup\{ \pm \infty\} . \mathrm{R}_{+}:=\{t \in \mathrm{R}: t \geq 0\}$.

For an arbitrary function $\varphi: E \rightarrow \overline{\mathrm{R}}$, the convex and concave conjugate function are denoted by $\varphi^{*}$ and $\varphi_{*}$, respectively; they are defined by $\varphi^{*}(y):=$ $\sup \{\langle y, x\rangle-\varphi(x): x \in E\}$ and $\left.\varphi_{*}(y):=\inf \{\langle y, x\rangle-\varphi(x): x \in E\}\right)$ [2]. Clearly, $-\varphi^{*}(\cdot)=(-\varphi)_{*}(-\cdot)$ and $-\varphi_{*}(\cdot)=(-\varphi)^{*}(-\cdot)$.

The biconjugation $\varphi^{* *}$ is defined by $\varphi^{* *}:=\left(\varphi^{*}\right)^{*}[2]$. It is well known that $\varphi^{* *} \leq \varphi$.

For an arbitrary subset $A$, the indicator function $\delta_{A}: E \rightarrow \overline{\mathrm{R}}$ is equals to 0 on $A$, and $+\infty$ otherwise. The associated convex and the concave support function $\delta_{A}^{+}$and $\delta_{A}^{-}: E \rightarrow \overline{\mathrm{R}}$ are defined by $\delta_{A}^{+}(v):=\sup _{w \in A}\langle v, w\rangle$ and $\delta_{A}^{-}(w):=\inf _{v \in A}\langle v, w\rangle$. Clearly, $\delta_{A}^{+}=\left(\delta_{A}\right)^{*}$ and $\delta_{A}^{-}=\left(-\delta_{A}\right)_{*}=-\left(\delta_{-A}\right)^{*}$. 
Finally, recall epi $\varphi:=\{(x, t) \in E \times \mathrm{R}: \varphi(x) \leq t\}$ and $B_{\varepsilon}(x):=\left\{x^{\prime} \in\right.$ $\left.E:\left\|x-x^{\prime}\right\|<\varepsilon\right\}$.

\section{Proof of the main theorem}

For every closed affine subspace $F$ of a Hilbert space $E$, the closed vector space $\vec{F}$ associated to $F$ is defined by $\vec{F}:=F-e_{F}$.

Lemma 1.1. Let $H$ be a closed affine hyperplane of $E$ with $\left\|e_{H}\right\| \neq 0$ and let $X$ be a nonempty convex set. Then

(4) $\inf _{x \in X} \sup _{y \in H}\langle x, y\rangle=\left\|e_{H}\right\|^{2} \inf \left\{\lambda \in \mathrm{R}: \lambda e_{H} \in X\right\}$,

(5) $\inf _{x \in X} \sup _{y \in H}\langle x, y\rangle<+\infty$ if and only if $X \cap R e_{H} \neq \emptyset \quad$ (inf-feasibility),

(6) $\sup _{y \in H} \inf _{x \in X}\langle x, y\rangle>-\infty$ if and only if $\quad-e_{H} \notin \bar{X}^{\infty} \quad$ (sup-feasibility).

Proof. To prove (4) observe that $H=\vec{H}+e_{H}$ and $\vec{H}^{\perp}=\mathrm{R} e_{H}$. Hence

$$
\begin{aligned}
\inf _{x \in X} \sup _{y \in H}\langle x, y\rangle & =\inf _{x \in X} \sup _{w \in H}\left\langle x, w+e_{H}\right\rangle=\inf _{x \in X} \sup _{w \in H}\left\{\langle x, w\rangle+\left\langle x, e_{H}\right\rangle\right\} \\
& =\inf \left\{\left\langle x, e_{H}\right\rangle: x \in X \cap \vec{H}^{\perp}\right\}=\inf \left\{\left\langle x, e_{H}\right\rangle: x \in X \cap \operatorname{Re}_{H}\right\} \\
& =\left\|e_{H}\right\|^{2} \inf \left\{\lambda \in \mathbf{R}: \lambda e_{H} \in X\right\} .
\end{aligned}
$$

Obviously, from (4) follows (5).

$\stackrel{(6)}{\Longrightarrow}$. When $\sup _{y \in H} \inf _{x \in X}\langle x, y\rangle>-\infty$, there exists $\bar{y} \in H$ with $\inf _{x \in X}\langle x, \bar{y}\rangle>-\infty$. Fix $\bar{x} \in X$. Since $\left\langle\bar{x}-t e_{H}, \bar{y}\right\rangle=\langle\bar{x}, \bar{y}\rangle-t\left\|e_{H}\right\|^{2}$, we have $\inf \left\{\langle x, \bar{y}\rangle: x \in \bar{x}-\mathbf{R}_{+} e_{H}\right\}=-\infty$. Therefore $\bar{x}-\mathbf{R}_{+} e_{H} \not \subset \bar{X}$. Thus $-e_{H} \notin \bar{X}^{\infty}$.

$\stackrel{(6)}{\Longleftarrow}$. Fix $\bar{x} \in X$. From $-e_{H} \notin \bar{X}^{\infty}$ it follows that there exists a real number $\bar{t}>0$ such that $\bar{x}-\bar{t} e_{H} \notin \bar{X}$. Hence, there is a closed hyperplane strictly separating $\bar{x}-\bar{t} e_{H}$ from $\bar{X}$; that is, there is a nonzero $\bar{w} \in E$ such that $\left\langle\bar{x}-\bar{t} e_{H}, \bar{w}\right\rangle<\langle x, \bar{w}\rangle$ for all $x \in X$. In particular, we have $\left\langle\bar{x}-\bar{t} e_{H}, \bar{w}\right\rangle<$ $\langle\bar{x}, \bar{w}\rangle$; consequently $\left\langle e_{H}, \bar{w}\right\rangle>0$. Now define $\bar{y}:=\frac{\left\|e_{H}\right\|^{2}}{\left\langle e_{H}, \bar{w}\right\rangle} \bar{w}$. Observe that $\bar{y} \in H$ and $\inf _{x \in X}\langle x, \bar{y}\rangle \geq\left\langle\bar{x}-\bar{t} e_{H}, \bar{y}\right\rangle$. Therefore $\sup _{y \in H} \inf _{x \in X}\langle x, y\rangle \geq$ $\inf _{x \in X}\langle x, \bar{y}\rangle>-\infty$.

Proof of Theorem 0.1. Since $F$ is a closed affine hyperplane of the Hilbert space $F_{0}:=\vec{F}+\operatorname{R} e_{F}$, we will use Lemma 1.1, replacing $H$ and $E$ with $F$ and $F_{0}$, respectively. 
For every $(x, y) \in X \times F$ we have $\langle x, y\rangle=\left\langle\operatorname{pr}_{0} x, y\right\rangle$. Hence,

$$
\begin{aligned}
\inf _{x \in X} \sup _{y \in F}\langle x, y\rangle & =\inf _{x \in \mathrm{pr}_{0}(X)} \sup _{y \in F}\langle x, y\rangle \\
& \stackrel{(\mathrm{i})}{=}\left\|e_{F}\right\|^{2} \inf \left\{\lambda \in \mathrm{R}: \lambda e_{F} \in \operatorname{pr}_{0}(X)\right\} \\
& \stackrel{(\dagger)}{\geq}\left\|e_{F}\right\|^{2} \inf \left\{\lambda \in \mathrm{R}: \lambda e_{F} \in \overline{\operatorname{pr}_{0}(X)}\right\} \stackrel{(\mathrm{i})}{=} \underset{x \in \inf _{0}(X)}{\sup }\langle x \in F \\
& \stackrel{(\ddagger)}{\geq} \sup _{y \in F} \inf _{x \in \operatorname{pr}_{0}(X)}\langle x, y\rangle \stackrel{(\mathrm{ii})}{=} \sup _{y \in F} \inf _{x \in \mathrm{pr}_{0}(X)}\langle x, y\rangle \\
& =\sup _{y \in F} \inf \langle x, y\rangle
\end{aligned}
$$

where equalities marked by (i) are due to (4) of Lemma 1.1, and equality (ii) follows from the continuity of the scalar product.

Part 1: necessity. Let $(X, F)$ be a minmax pair. Then inequalities $(\dagger)$ and $(\ddagger)$ in (a) become equalities. Therefore, (2) holds and inf ${ }_{x \in \overline{\operatorname{ro}_{0}(X)}} \sup _{y \in F}\langle x, y\rangle=$ $\sup _{y \in F} \inf _{x \in \overline{\operatorname{pr}_{0}(X)}}\langle x, y\rangle$. Hence,

either $\inf _{x \in \overline{\mathrm{pr}_{0}(X)}} \sup _{y \in F}\langle x, y\rangle<+\infty \quad$ or $\quad \sup _{y \in F} \inf _{x \in \overline{\mathrm{pr}}_{0}(X)}\langle x, y\rangle>-\infty$;

therefore (3) holds, in virtue of (5)-(6) of Lemma 1.1.

Part 2: sufficiency. Assume (2) and (3) hold. Thus, inequality ( $\dagger$ ) in (a) becomes an equality. By (a), to complete the proof it is enough to show that

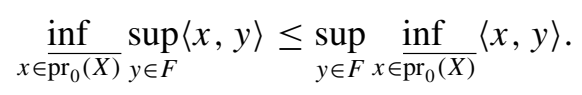

Consider the following two cases:

$1^{\text {st }}$ case: " $\overline{\mathrm{pr}_{0}(X)} \cap \mathrm{R} e_{F} \neq \emptyset$ ". Choose a real number $\bar{\lambda}$ such that $\bar{\lambda} e_{F} \in$ $\overline{\operatorname{pr}_{0}(X)}$. Let $a$ be a real number such that $a<\inf _{x \in \overline{\mathrm{r}_{0}(X)}} \sup _{y \in F}\langle x, y\rangle$. By (4) of Lemma 1.1 we have $a<\left\|e_{F}\right\|^{2} \inf \left\{\lambda \in \mathrm{R}: \lambda e_{F} \in \overline{\operatorname{pr}_{0}(X)}\right\}$. Therefore,

$$
\frac{a}{\left\|e_{F}\right\|^{2}} e_{F} \notin \overline{\operatorname{pr}_{0}(X)} \quad \text { and } \quad a<\left\|e_{F}\right\|^{2} \bar{\lambda} \text {. }
$$

Strictly separating the point $\frac{a}{\left\|e_{F}\right\|^{2}} e_{F}$ from $\overline{\operatorname{pr}_{0}(X)}$ by a closed hyperplane of $F_{0}$, we get a nonzero $\bar{w} \in F_{0}$ such that

$$
\left\langle\frac{a}{\left\|e_{F}\right\|^{2}} e_{F}, \bar{w}\right\rangle<\langle x, \bar{w}\rangle \quad \text { for all } \quad x \in \overline{\operatorname{pr}_{0}(X)} .
$$


In particular, we have $\left\langle\frac{a}{\left\|e_{F}\right\|^{2}} e_{F}, \bar{w}\right\rangle<\left\langle\bar{\lambda} e_{F}, \bar{w}\right\rangle$; consequently $\left\langle e_{F}, \bar{w}\right\rangle>0$. Now define $\bar{y}:=\frac{\left\|e_{F}\right\|^{2}}{\left\langle e_{F}, \bar{w}\right\rangle} \bar{w}$. Observe that $\bar{y} \in F$ and by $(c)$ we have

$$
\inf _{x \in \overline{\operatorname{pr}_{0}(X)}}\langle x, \bar{y}\rangle \geq\left\langle\frac{a}{\left\|e_{F}\right\|^{2}} e_{F}, \bar{y}\right\rangle=a .
$$

Therefore $\sup _{y \in F} \inf _{x \in \overline{\operatorname{pr}_{0}(X)}}\langle x, y\rangle \geq \inf _{x \in \overline{\mathrm{pr}_{0}(X)}}\langle x, \bar{y}\rangle \geq a$. As $a$ is an arbitrary real number such that $a<\inf _{x \in \overline{\mathrm{pr}_{0}(X)}} \sup _{y \in F}\langle x, y\rangle$, inequality (b) holds.

$$
2^{\text {nd }} \text { case: “ }-e_{F} \notin{\overline{\operatorname{pr}_{0}(X)}}^{\infty} \text { and } \overline{\operatorname{pr}_{0}(X)} \cap \operatorname{Re}_{F}=\emptyset ” \text {. By (5) and (6) of }
$$
Lemma 1.1 we have

$$
\inf _{x \in \overline{\operatorname{pr}_{0}(X)}} \sup _{y \in F}\langle x, y\rangle=+\infty \quad \text { and } \quad \sup _{y \in F} \underset{x \in \overline{\operatorname{pr}_{0}(X)}}{\inf }\langle x, y\rangle>-\infty,
$$

respectively. Therefore, there exist $\bar{y} \in F$ and $M \in \mathrm{R}$ such that

$$
\langle x, \bar{y}\rangle \geq M \quad \text { for all } \quad x \in \overline{\operatorname{pr}_{0}(X)} .
$$

Fix a real number $a$. The closed (locally compact) ray $K:=\left\{\lambda e_{F}: \lambda \in \mathrm{R}, \lambda \leq\right.$ $a\}$ and the closed convex set $\overline{\operatorname{pr}_{0}(X)}$ are disjoint and the intersection of their asymptotic cones reduces to the zero vector. Hence, by Dieudonné's separation theorem (see [4, Theorem 15D]), $K$ and $\overline{\operatorname{pr}_{0}(X)}$ are strictly separated by an hyperplane of $F_{0}$; that is, there exist a nonzero $\bar{w} \in F_{0}$ and a real number $\mu$ such that

(e) $\left\langle t e_{F}, \bar{w}\right\rangle<\mu<\langle x, \bar{w}\rangle \quad$ for every $\quad x \in \overline{\operatorname{pr}_{0}(X)}$ and $t \leq a$.

Since $\overline{\operatorname{pr}_{0}(X)} \neq \emptyset$, we have $\left\langle e_{F}, \bar{w}\right\rangle \geq 0$. Consider the following two subcases. First sub-case: " $\left\langle e_{F}, \bar{w}\right\rangle>0$ ". Reason as in the first case to complete the proof. Second sub-case: " $\left\langle e_{F}, \bar{w}\right\rangle=0$ ". Since $\bar{y} \in F$, the point $y_{t}:=\bar{y}+t \bar{w}$ belongs to $F$ for every real number $t$. From (e) it follows that $\mu>0$. Hence (d) and (e) imply that for all $x \in \overline{\operatorname{pr}_{0}(X)}$ and $t \in \mathrm{R}$

$$
\left\langle x, y_{t}\right\rangle=\langle x, \bar{y}\rangle+t\langle x, \bar{w}\rangle \geq M+t \mu .
$$

Therefore

$$
\sup _{y \in F} \underset{x \in \overline{\operatorname{pr}_{0}(X)}}{\inf }\langle x, y\rangle \geq \sup _{t \in \mathrm{R}} \underset{x \in \overline{\operatorname{pr}_{0}(X)}}{\inf }\left\langle x, y_{t}\right\rangle \geq \sup _{t \in \mathrm{R}}(M+t \mu)=+\infty .
$$

Thus, (b) holds. 


\section{Fenchel equalities and minmax convex pairs}

Upon generalizing key properties between pairs of linear programs to nonlinear problems, Fenchel [2, Chap. III, §5-6] founded the duality theory of convex optimization, thereby bringing to the fore the chief roles of conjugation

$\varphi^{*}(y):=\sup \{\langle y, x\rangle-\varphi(x): x \in E\}, \quad \varphi_{*}(y):=\inf \{\langle y, x\rangle-\varphi(x): x \in E\}$, and inf-convolution

$$
\varphi \nabla \psi(w):=\inf _{x \in E}\{\varphi(x)+\psi(w-x)\} .
$$

Prominent in his approach are pairs $(\varphi, \psi)$ of convex functions $\varphi, \psi: E \rightarrow \overline{\mathbf{R}}$ satisfying

$$
\inf _{x \in E}\{\varphi(x)+\psi(x)\}=\sup _{y \in E}\left\{(-\varphi)_{*}(y)+(-\psi)_{*}(-y)\right\}
$$

When valid, (7) will be referred to as a Fenchel equality with $(\varphi, \psi)$ as a Fenchel pair, and assertions about such facts are briefly called null duality gap theorems. Whenever some extremum in (7) is attained, the result is known as a Fenchel duality theorem; see [1, Theorem 3.3.5, p. 52 and Corollary 5.1.9, p. 100].

A first appearance of such theorems is due to Fenchel; see Propositions 47 and 48 in [2, Ch. III §6]. Fenchel's Proposition 47: "A couple $(\varphi, \psi)$ of convex functions is a Fenchel pair, if $\varphi$ and $\psi$ are lower semi-continuous proper convex functions and are finite in a same point" is not well stated. For guarantying its validity some supplementary assumptions must be recovered from Fenchel's proofs; this will be done in next Theorem 3.1, via (14) and (16).

Further, in Proposition 49 of [2, Ch. III $\S 6$, p. 110] Fenchel restated the said Proposition 47, concerning null duality gap, as a minmax equality with respect to the convex-concave bivariate function

$$
(x, y) \mapsto\langle x, y\rangle+\varphi(x)-\psi^{*}(y) .
$$

He noticed that for $\varphi, \psi$ proper and $\psi$ lower semi-continuous one has

$$
\begin{aligned}
& \inf _{x \in E} \sup _{y \in E}\left\{\langle x, y\rangle+\varphi(x)-\psi^{*}(y)\right\}=\inf _{x \in E}\{\varphi(x)+\psi(x)\} \\
& \sup _{y \in E} \inf _{x \in E}\left\{\langle x, y\rangle+\varphi(x)-\psi^{*}(y)\right\}=\sup _{y \in E}\left\{(-\varphi)_{*}(y)+\left(-\psi_{*}\right)(-y)\right\} .
\end{aligned}
$$

Subsequently, Fenchel [2, Ch. III §6, p. 112] used his duality theorem to derive von Neumann's bilinear minmax theorem, applying (8) to the case where 
$\varphi$ and $\psi^{*}$ are extended indicators of simplices. Following Fenchel's approach, we observe that when $X, Y$ both are nonempty convex,

$$
(X, \bar{Y}) \text { is a minmax pair } \Longleftrightarrow\left(\delta_{X}, \delta_{Y}^{+}\right) \text {is a Fenchel pair. }
$$

On the other hand,

(10) $(X, Y)$ is a minmax pair $\Longleftrightarrow(X, \bar{Y})$ and $(\bar{X}, Y)$ are minmax pairs;

in fact $\sup _{Y} \inf _{X}\langle.,\rangle=.\sup _{Y} \inf _{\bar{X}}\langle.,.\rangle \leq \sup _{\bar{Y}} \inf _{X}\langle.,.\rangle \leq \inf _{\bar{X}} \sup _{Y}\langle.,$. $\inf _{X} \sup _{Y}\langle.,\rangle=.\inf _{X} \sup _{\bar{Y}}\langle.,$.$\rangle . Thus, by (9) the notion of Fenchel pair$ provides a criterion for recognizing minmax pairs:

$(X, Y)$ is a minmax pair $\Longleftrightarrow\left(\delta_{X}, \delta_{Y}^{+}\right)$and $\left(-\delta_{X}^{-}, \delta_{Y}\right)$ are Fenchel pairs.

This tells that Fenchel equalities encompass minmax pairs. Conversely, minmax convex pairs capture both biconjugation formulae and Fenchel equalities. This will become clear from (12)-(16) below.

(12) (Fenchel biconjugation formula and minmax pairs) Let $\varphi: E \rightarrow \overline{\mathrm{R}}$ be a convex function. Define

$$
H:=\{(y, t) \in E \times \mathrm{R}: y \in E, t=1\} .
$$

Then $H$ is a closed affine hyperplane of the Hilbert space $E \times \mathrm{R}$ and

$$
\varphi^{* *}(0)=\varphi(0) \Longleftrightarrow(\text { epi } \varphi, H) \text { is a minmax pair. }
$$

In short, $\varphi: E \rightarrow \overline{\mathrm{R}}$ is said to be Fenchel regular at 0 , if $\varphi^{* *}(0)=\varphi(0)$. A function $\varphi$ is said to be Fenchel regular if $\varphi^{* *}=\varphi$. Fenchel showed that lower-semicontinuous proper convex functions are regular $[2, \mathrm{Ch}$. III §37, p. 91]

(13) (Fenchel equality and minmax pairs) Let $\varphi, \psi: E \rightarrow \overline{\mathrm{R}}$ be convex functions. Let $H$ be as in (12) and

$$
F:=\left\{(y, 1,-y, 1) \in(E \times \mathbf{R})^{2}: y \in E\right\} .
$$

Then $F$ is a closed affine subspace of the Hilbert space $(E \times \mathrm{R})^{2}$ and

$(\varphi, \psi)$ is a Fenchel pair

$\Longleftrightarrow($ epi $\varphi \times$ epi $\psi, F)$ is a minmax pair

$\Longleftrightarrow($ epi $\varphi+\operatorname{epi}(\psi \circ \sigma), H)$ is a minmax pair

where $\sigma: E \rightarrow E$ defined be $\sigma(y):=-y$. 
(14) (Fenchel inf-convolution formula and minmax pairs) In [2, Ch. III §5, proof of Prop. 47 p. 106] Fenchel used the inf-convolution formula " $\varphi^{*} \nabla \psi^{*}(0)=(\varphi+\psi)^{*}(0)$ ". Observe that

$$
\begin{aligned}
\varphi^{*} \nabla \psi^{*}(0) & =(\varphi+\psi)^{*}(0) \\
& \Longleftrightarrow(\text { epi } \varphi \times \text { epi } \psi, F) \text { is a minmax pair } \\
\Longleftrightarrow & (\text { epi } \varphi+\operatorname{epi}(\psi \circ \sigma), H) \text { is a minmax pair, }
\end{aligned}
$$

for every $\varphi, \psi: E \rightarrow \overline{\mathrm{R}}$ convex functions with epi $\varphi \neq \emptyset$ and epi $\psi \neq \emptyset$, and $H, F$ and $\sigma$ are as in (13).

(15) (Moreau inf-convolution formula and minmax pairs) In [5, §9.a, p. 56] Moreau investigated the inf-convolution formula " $\varphi \nabla \psi(0)=\left(\varphi^{*}+\right.$ $\left.\psi^{*}\right)^{*}(0)$ ". Observe that

$$
\begin{aligned}
\varphi \nabla \psi(0)=\left(\varphi^{*}+\psi^{*}\right)^{*}(0) & \Longleftrightarrow\left(\text { epi } \varphi \times \text { epi } \psi, F^{\prime}\right) \text { is a minmax pair } \\
& \Longleftrightarrow(\text { epi } \varphi+\text { epi } \psi, H) \text { is a minmax pair, }
\end{aligned}
$$

where $\varphi, \psi, H$ are as above in (14) and $F^{\prime}$ is a closed affine subspace of the Hilbert space $(E \times \mathrm{R})^{2}$ which is defined by

$$
F^{\prime}:=\left\{(y, 1, y, 1) \in(E \times \mathrm{R})^{2}: y \in E\right\} .
$$

(16) (Fenchel regularity of inf-convolution and minmax pairs) Let $\varphi, \psi$ : $E \rightarrow \overline{\mathrm{R}}$ be convex functions. Let $H$ be as in (12). Then

$$
(\varphi \nabla \psi)^{* *}(0)=(\varphi \nabla \psi)(0) \Longleftrightarrow(\text { epi } \varphi+\text { epi } \psi, H) \text { is a minmax pair. }
$$

Together observations (12)-(16) show that the concept of minmax convex pairs unifies much of convex duality theory. It is noteworthy that as basic bivariate function we invoked merely the inner scalar product. Also noteworthy is the importance of instances in which the second set of the minmax pair is an affine closed subspace or an hyperplane. This last observation motivated us to give Theorem 0.1: a "geometric characterization" of such instances.

\section{Concluding Comments}

To illustrate the topological significance of properties (2) and (3) we apply Theorem 1 to Fenchel equalities and to biconjugation formulae.

Taking into account (13), we have a basic "null duality gap theorem" whose sketched out proof provides a topological insight into (2) and (3). 
THEOREM 3.1 (Null duality gap). Let $\varphi, \psi: E \rightarrow \overline{\mathrm{R}}$ arbitrary convex functions. Then $(\varphi, \psi)$ is a Fenchel pair if and only if the following properties hold:

$$
\begin{aligned}
& \text { (17) } \inf _{x \in E}\{\varphi(x)+\psi(x)\} \leq \liminf _{v \rightarrow 0} \inf _{x \in E}\{\varphi(x)+\psi(x+v)\} \quad \text { (inf-normality) } \\
& \text { (18) either } \inf _{x \in E}\{\varphi(x)+\psi(x)\}<+\infty \\
& \text { or } \sup _{y \in E}\left\{(-\varphi)_{*}(y)+(-\psi)_{*}(-y)\right\}>-\infty \quad \text { (feasibility). }
\end{aligned}
$$

Proof. Without loss of generality assume epi $\varphi \neq \emptyset$ and epi $\psi \neq \emptyset$. Set $\left(1^{*}\right)$

$$
X:=\operatorname{epi} \varphi \times \text { epi } \psi \quad \text { and } \quad F:=\left\{(y, 1,-y, 1) \in(E \times \mathrm{R})^{2}: y \in E\right\} .
$$

Clearly $X$ is a nonempty convex set and $F$ is a closed affine subspace of the Hilbert space $(E \times \mathbf{R})^{2}$. We have

$\left(3^{*}\right) \quad F_{0}=\{(y, \lambda,-y, \lambda): y \in E, \lambda \in \mathrm{R}\}$

$\left(4^{*}\right) \quad \operatorname{pr}_{0}\left(x, t, x^{\prime}, t^{\prime}\right)=\frac{1}{2}\left(x-x^{\prime}, t+t^{\prime}, x^{\prime}-x, t+t^{\prime}\right)$

$$
\text { for all }\left(x, t, x^{\prime}, t^{\prime}\right) \in(E \times \mathrm{R})^{2}
$$

$\left(5^{*}\right) \quad \inf \left\{\lambda \in \mathrm{R}: \lambda e_{F} \in \operatorname{pr}_{0} X\right\}=\frac{1}{2} \inf _{x \in E}\{\varphi(x)+\psi(x)\}$

$\left(6^{*}\right) \quad \inf \left\{\lambda \in \mathrm{R}: \lambda e_{F} \in \overline{\operatorname{pr}_{0} X}\right\}=\frac{1}{2} \liminf _{v \rightarrow 0} \inf _{x \in E}\{\varphi(x)+\psi(x+v)\}$

$\left(7^{*}\right) \quad \overline{\operatorname{pr}_{0} X} \cap \operatorname{Re} e_{F} \neq \emptyset \Longleftrightarrow \liminf _{v \rightarrow 0} \inf _{x \in E}\{\varphi(x)+\psi(x+v)\}<+\infty$

$$
-e_{F} \notin{\overline{\mathrm{pr}_{0} X}}^{\infty} \Longleftrightarrow \sup _{y \in E}\left\{(-\varphi)_{*}(y)+(-\psi)_{*}(-y)\right\}>-\infty .
$$

Hence, from (13) and Theorem 0.1 the required equivalence follows.

If the affine subspace $F$ in Theorem 0.1 is an hyperplane, the orthogonal projection $\mathrm{pr}_{0}$ is the identity of $E$. Therefore

Theorem 3.2 (Minmax convex pairs and affine hyperplanes). Let $H$ be a closed affine hyperplane of $E$ with $\left\|e_{H}\right\| \neq 0$. Let $X$ be a nonempty convex 
subset of $E$. Then $(X, H)$ is a minmax pair if and only if the following two conditions hold:

(20) either $\bar{X} \cap \mathrm{R} e_{H} \neq \emptyset$ or $-e_{H} \notin \bar{X}^{\infty}$

(inf-normality),

(feasibility).

Hence, from (12) we have the following (known) basic criterion for biconjugation formulae.

TheOREM 3.3 (Biconjugation formula). Let $\varphi: E \rightarrow \overline{\mathrm{R}}$ an arbitrary convex function. Then

$$
\varphi^{* *}(0)=\varphi(0) \Longleftrightarrow \begin{cases}\text { (i) } & \varphi \text { is lower semicontinuous at } 0 \\ \text { (ii) } & \text { either } \varphi(0)<+\infty \text { or } \varphi^{* *}(0)>-\infty\end{cases}
$$

Proof. Without loss of generality assume epi $\varphi \neq \emptyset$. Set

$$
X:=\operatorname{epi} \varphi \quad \text { and } \quad H:=\{(y, 1) \in E \times \mathrm{R}: y \in E\} .
$$

Clearly $X$ is a nonempty convex set and $H$ is closed affine hyperplane of the Hilbert space $E \times \mathrm{R}$. We have

$$
\begin{aligned}
& e_{H}=(0,1) \\
& \inf \left\{\lambda \in \mathrm{R}: \lambda e_{F} \in X\right\}=\varphi(0) \\
& \quad \text { and } \quad \inf \left\{\lambda \in \mathrm{R}: \lambda e_{F} \in \bar{X}\right\}=\liminf _{x \rightarrow 0} \varphi(x) \\
& \bar{X} \cap \operatorname{R} e_{H} \neq \emptyset \Longleftrightarrow \liminf _{x \rightarrow 0} \varphi(x)<+\infty \\
& -e_{H} \notin \bar{X}^{\infty} \Longleftrightarrow \varphi^{* *}(0)>-\infty .
\end{aligned}
$$

Hence, from (12) and Theorem 0.1 the required equivalence follows.

Theorem 3.4 (A criterion for minmax convex pairs [3]). A couple (X, $Y$ ) of nonempty convex sets is a minmax pair if and only if the following properties hold:

(22) $\inf _{x \in X} \sup _{y \in Y}\langle x, y\rangle \leq \lim _{\varepsilon \rightarrow 0} \inf _{x \in B_{\varepsilon}(X)} \sup _{y \in Y}\langle x, y\rangle$

(inf-normality)

(23) either $\inf _{x \in X} \sup _{y \in Y}\langle x, y\rangle<+\infty$ or $\sup _{y \in Y} \inf _{x \in X}\langle x, y\rangle>-\infty \quad$ (feasibility) (24) $\sup _{y \in \bar{Y}} \inf _{x \in X}\langle x, y\rangle=\sup _{y \in Y} \inf _{x \in X}\langle x, y\rangle \quad$ (boundary sup-negligibility). 
Proof. First, observe that

$$
\sup _{y \in Y} \inf \langle x, y\rangle \leq \sup _{x \in X} \inf \langle x, y\rangle \leq \inf _{x \in X} \sup _{x \in X}\langle x, y\rangle=\inf _{x \in X} \sup _{y \in Y}\langle x, y\rangle .
$$

Define $\varphi, \psi: E \rightarrow \overline{\mathrm{R}}$ by $\varphi:=\delta_{X}$ and $\psi:=\delta_{Y}^{+}$. Then

$$
\inf _{x \in X} \sup _{y \in Y}\langle x, y\rangle=\inf _{x \in E}\left\{\delta_{X}(x)+\delta_{Y}^{+}(x)\right\} .
$$

Moreover, since $\left(-\delta_{X}\right)_{*}(y)=\delta_{X}^{-}(y)$ and $\left(-\delta_{Y}^{+}\right)_{*}(-y)=\delta_{\bar{Y}}(y)$, we have

$$
\sup _{y \in E}\left\{\left(-\delta_{X}\right)_{*}(y)+\left(-\delta_{Y}^{+}\right)_{*}(-y)\right\}=\sup _{\bar{Y}} \inf \langle x, y\rangle .
$$

Conditions (17) and (18) become (22) and (23), respectively. Hence, from Theorem 3.1 it follows that

$$
(22) \&(23) \Longleftrightarrow \inf _{x \in E}\left\{\delta_{X}(x)-\delta_{Y}^{+}(x)\right\}=\sup _{y \in E}\left\{\left(-\delta_{X}\right)_{*}(y)+\left(-\delta_{Y}^{+}\right)_{*}(-y)\right\} .
$$

Therefore, combining $\left(1^{*}\right)-\left(4^{*}\right)$, we have the required equivalence.

Similarly, (11) yields the following criterion.

Theorem 3.5 (A criterion for minmax convex pairs [3]). A couple $(X, Y)$ of nonempty convex sets is a minmax pair if and only if (22), (23) and the following property hold:

$$
\sup _{y \in Y} \inf \langle x, y\rangle \geq \lim _{\varepsilon \rightarrow 0} \sup _{y \in B_{\varepsilon}(Y)} \inf _{x \in X}\langle x, y\rangle \quad \text { (sup-normality). }
$$

The previous six theorems $0.1,3.1-3.5$ are "logically equivalent"; that is, any one of them "implies" the other five. For example

(26) Theorem 3.1 is derivable from Theorem 3.3 by observing that for the optimal value function $\gamma: E \rightarrow \overline{\mathrm{R}}$ (defined by $\gamma(v):=\inf _{x \in E}\{\varphi(x)+$ $\psi(x+v)\})$ one has: $\gamma(0)=\inf _{x \in E}\{\varphi(x)+\psi(x)\}$ and $\gamma^{* *}(0)=$ $\sup _{y \in E}\left\{(-\varphi)_{*}(y)+(-\psi)_{*}(-y)\right\}$.

(27) Theorem 3.2 is derivable from Theorem 3.3 by observing that for the function $\varphi_{X}: \vec{H} \rightarrow \overline{\mathrm{R}}$ which is defined by $\varphi_{X}(v):=\inf \left\{\left\langle x, e_{H}\right\rangle: x \in\right.$ $\left.X \cap\left(v+\operatorname{R} e_{H}\right)\right\}$, one has: $\varphi_{X}^{* *}(0)=\sup _{y \in H} \inf _{x \in X}\langle x, y\rangle \leq$ $\inf _{x \in X} \sup _{y \in H}\langle x, y\rangle=\varphi_{X}(0)$.

(28) Theorem 3.4 is derivable from Theorem 3.3 by observing that for the function $\varphi: E \rightarrow \overline{\mathrm{R}}$ which is defined by $\varphi(v):=\inf _{x \in X} \sup _{y \in Y}\langle x+$ 
$v, y\rangle$, one has: $\inf _{v \in B_{\varepsilon}(0)} \varphi(v)=\inf _{x \in B_{\varepsilon}(X)} \sup _{y \in Y}\langle x, y\rangle$ and $\varphi^{* *}(0)=$ $\sup _{y \in \bar{Y}} \inf _{x \in X}\langle x, y\rangle \leq \inf _{x \in X} \sup _{y \in \bar{Y}}\langle x, y\rangle=\varphi(0)$.

Two extremely simplified "equivalent reformulations" of the previous theorems are given by the following theorems.

THEOREM 3.6 (Minmax pairs of closed convex sets and affine hyperplanes). Let $H$ be a closed affine hyperplane of $E$ with $\left\|e_{H}\right\| \neq 0$. For every nonempty closed convex set $X$ :

(29) $(X, H)$ is a minmax pair $\Longleftrightarrow$ either $X \cap R e_{H} \neq \emptyset$ or $-e_{H} \notin X^{\infty}$.

THEOREM 3.7 (Biconjugation formula). Let $\varphi: E \rightarrow \overline{\mathrm{R}}$ a lower-semicontinuous convex function. Then

$$
\varphi^{* *}(0)=\varphi(0) \Longleftrightarrow \text { either } \varphi(0)<+\infty \text { or } \varphi^{* *}(0)>-\infty \text {. }
$$

\section{REFERENCES}

1. Borwein, J. M., Lewis, A. S., Convex Analysis and Nonlinear Optimization, Theory and Examples, Springer-Verlag, New York, 2000.

2. Fenchel, W., Convex Cones, Sets, and Functions, mimeographed lecture notes, Princeton Univ. Press, 1951.

3. Greco, G. H., Minmax convex pairs, J. Convex Anal. 13 (2006), in press.

4. Holmes, R. B., Geometric Functional Analysis and its Applications, Springer-Verlag, New York, 1975.

5. Moreau, J. J., Fonctionnelles convexes, mimeographed lecture notes, Collège de France, 1966-67.

6. von Neumann, J., Zur Theorie der Gesellschaftsspiele, Math. Ann. 100 (1928), 295-320.

DIPARTIMENTO DI MATEMATICA

UNIVERSITÀ DI TRENTO

38050 POVO

ITALY

E-mail: greco@science.unitn.it
UNIVERSIDAD DE TARAPACÁ DEPARTAMENTO DE MATEMÁTICA

CASILLA 7D

ARICA

CHILE

E-mail: aflores@uta.cl,hroman@uta.cl 